\title{
The Measurement Bench for the LHC Spool Corrector Magnets in Industry
}

\author{
A. Arn, S. A. Arshad, C. Giloux, F. Patru, H. Reymond, R. Senis, and L. Walckiers
}

\begin{abstract}
The LHC accelerator will be equipped with more than 3500 superconducting spool corrector magnets. CERN has awarded the contract for the series production and testing of these corrector magnets to industry. Magnetic field measurements are done at the factory.

Dedicated magnetic measurement benches have been built to test these corrector magnets in the resistive state at room temperature. The benches allow to measure the strength of the main field, normal and skew harmonics, the magnetic axis position and orientation of the main field with respect to the mechanical reference points of the magnet.

This paper presents the objectives, a description and the performances obtained with the benches during first measurements at industry.
\end{abstract}

Index Terms-Corrector, magnetic field measurement, superconducting magnet.

\section{INTRODUCTION}

$\mathbf{T}$ HE MAIN dipoles (MB) of LHC will be equipped with Sextupole (MCS) and Decapole (MCD) "spool" correctors. Each decapole corrector has in addition an Octupole insert (MCO) and these together are designated as MCDO. In total $2464 \mathrm{MCS}$ and $1232 \mathrm{MCDO}$ assemblies are required for the LHC. Half of these will be made in India in a collaboration between CERN and CAT (Centre for Advanced Technology), the remainder will be built by two European firms, ANTEC, Spain and TESLA Engineering, UK. Pairs of MCS's and MCDO's magnets are mounted at each end of the MB's to correct field errors in the dipole field caused mainly by persistent currents in their superconducting cables (filament magnetization).

The measurements performed so far on the model corrector magnets allow to define the following strategy for the series measurements of the LHC correctors [1]. A good correlation has been obtained between measurements at nominal strength in superfluid helium and on a dedicated bench for measurements at room temperature [2]. The bench described here has been designed to routinely control at room temperature the production in the magnet manufacturer's premises before and after systematic tests of the quench performance of the magnets at $4.3 \mathrm{~K}$. We analyze the performance reached to measure the field quality, the strength of the magnet, and the position of the magnetic axis with respect to the mechanical reference points.

Manuscript received September 24, 2001.

The authors are with CERN, CH-1211 Geneva 23, Switzerland (e-mail: raphael.senis@cern.ch).

Publisher Item Identifier S 1051-8223(02)04265-3.

\section{THE Bench FOR RoOM TeMPeRATURE MAGNetiC MEASUREMENTS}

\section{A. Functional Description}

The quality of the magnetic field and magnetic axis alignment depends on the precision of the magnet coils and their assembly in the yoke and casing, difficult to measure on a finished magnet assembly. Therefore a relatively simple magnetic field measurement at room temperature is done by the manufacturer to obtain these data and confirmation that the assembly is within tolerance. This test also detects gross errors like inversely connected coils, coils with a missing turn or interturn short circuits. The field quality is recorded and an acceptance or out-of-tolerance indication obtained.

The CERN-built room temperature industry bench (Fig. 1) includes a mechanical bench on which the magnet is installed without demounting the rotating measurement coil, and a rack containing the VME integrators, the bipolar power supply, a Sun/Labview data acquisition system, and a printer to easily record the main parameters of each measured magnets.

The magnet, weighing about $8 \mathrm{~kg}$, is lowered manually down onto the bench and positioned with the reference holes on the magnet's mechanical casing. These holes are later used to position the magnets at the end of the MB dipoles within $0.1 \mathrm{~mm}$. A bipolar DC power supply feeds the magnet under test $(1 \mathrm{~A}$ for MCS and 0.5 A for MCDO), the nominal current of the magnets being $550 \mathrm{~A}$.

Installation and removal of the magnet typically last one minute and the automatic measurement procedure takes four minutes. Three MCS and three MCDO benches were built, and have now been installed in industry. The bench assembly and training of the operators was achieved in one day.

\section{B. Rotating Shaft Holding the Measuring Coils}

The measurement is based on the rotating coil principle [3]. This method consists of taking the values of the flux linked with the coil at (equally space) angular positions. The coil angular position is read by an angular encoder. The signal (flux as a function of angle) is obtained from the measured coil voltage during the coil rotation by integration.

The rotating shaft is equipped with a pair of symmetrically located single "outer" tangential coils, one serving as spare or for diagnostic purposes. The rotation radius and opening angle of the coils are maximized to measure harmonics up to order 15.

The bench is purposely open at the top to enable installation of the magnet without need to decouple the motor, encoder and shaft with respect to each other, so that the calibration of the bench is conserved. Slip rings add to robustness of operation. 


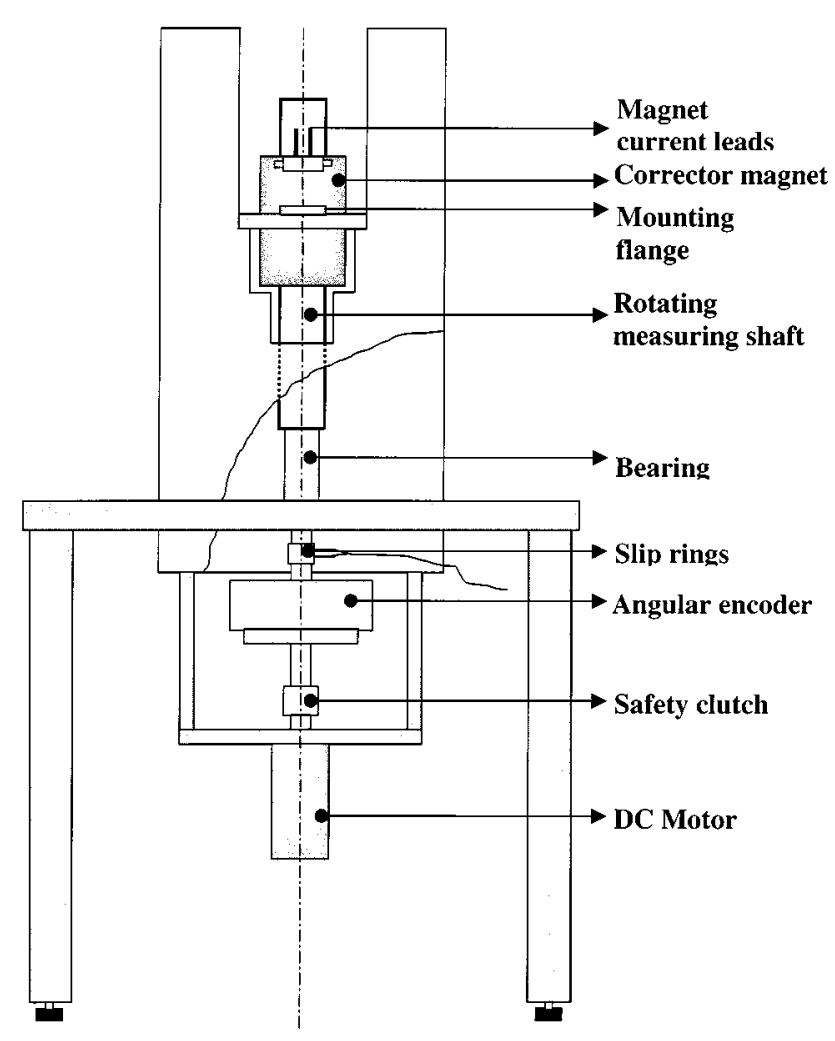

Fig. 1. Bench description for the room temperature magnetic measurement.

Due to the comfortable aspect ratio of the shaft (length/ diameter) and design of the bearings, vibrations are reduced so that no bucking coils are needed. The bearings are in selflubricating bronze. Their diameter and vertical spacing guarantee a mechanical stability of the top of the rotating shaft within 10 microns.

\section{Data Acquisition and Analysis}

Rotating coil based measurement [3] are repeated with both direction of rotation to correct for shaft twist and electronic drifts, and with both current polarities to correct for permanent magnetization and pick-up of external fields. Data are obtained from averages of 10 such measurement sequences. The results are then analyzed to generate the levels of the harmonics up to order 15 , the position of the magnetic axis and orientation of the field with respect to the reference holes. These measurements are then compared with the acceptable levels, and if the tolerance is exceeded anywhere it is highlighted on the screen. The LabView/Sun based MMP program [4] used for the magnetic measurement of all the LHC magnets has been tuned to reduce to a minimum the man machine interface.

\section{Locating the Magnetic Axis and Bench Calibration}

Rotating coils give the field multipoles in the reference frame of the rotation axis. The magnetic axis of a sextupole or decapole is defined as the line where respectively the quadrupole or octupole term vanishes [5]. LHC optics considerations require the magnetic axis of the correctors to be aligned to within $0.1 \mathrm{~mm}$ with respect to the ends of the MB main dipole magnets. The measurement gives the offset between two frames: the mea-

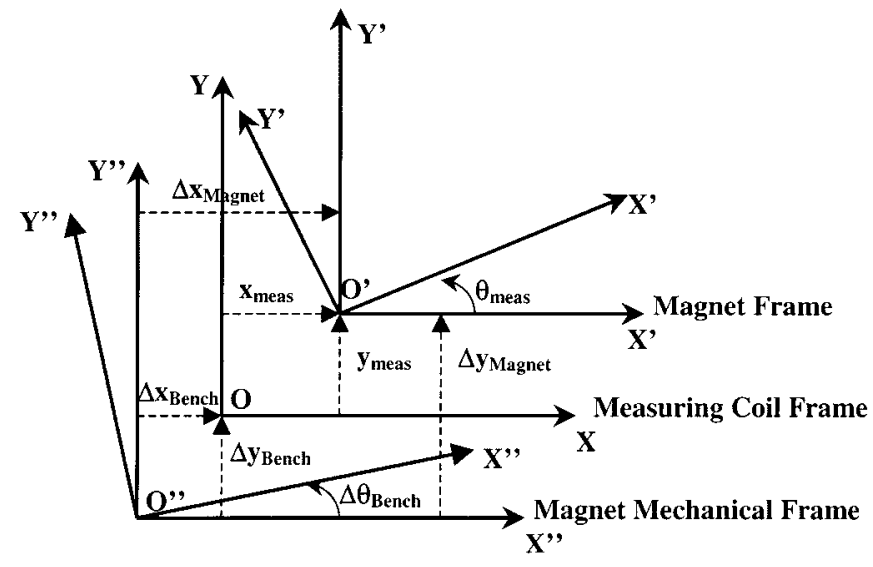

Fig. 2. Position of the measuring coil axis with respect to the magnet reference frame and the reference magnet's mechanical frame.

suring coil reference frame, $X-Y$, with the origin at the rotation axis passing through $O$ and the magnet reference frame, $X^{\prime}-Y^{\prime}$, with the origin at the magnetic axis passing through $O^{\prime}$, located at $\left(x_{\text {meas }}, y_{\text {meas }}\right)$ in the $X-Y$ frame (Fig. 2). The measured multipoles translated by $\left(x_{\text {meas }}, y_{\text {meas }}\right)$ can readily be calculated in the reference frame of the magnetic axis.

The magnet is positioned on the bench using the reference holes on the magnet's flange welded on the external case and used to position the magnet at the MB ends. All magnet components (especially coils) are produced with reference dowel holes symmetrically located at $70 \mathrm{~mm}$ from the magnet's axis. The positioning of the poles in the MCS or MCDO case is accurate enough such that mechanical axis, located by definition at $70 \mathrm{~mm}$ from one of the dowel holes in the magnet's case, and magnetic axis coincide within $0.1 \mathrm{~mm}$ [2].

The axis of rotation of the coils is not perfectly centered with respect to the dowel pins positioning the magnets on the bench. A calibration corrects the resulting systematic error. A reference magnet is measured in three different positions: rotated by $180^{\circ}$ with respect to the normal position to measure $X-Y$ offset $\left(\Delta x_{B e n c h}, \Delta y_{B e n c h}\right)$, and turned end to end to measure the angular offset $\left(\Delta \theta_{\text {Bench }}\right)$. In the reference magnet, we know precisely (metrology data) the length between the two dowel holes and thus the deviation $\varepsilon$, from the nominal value $(140 \mathrm{~mm})$. The LabView application calculates the three values $\left(\Delta x_{\text {Bench }}, \Delta y_{\text {Bench }}, \Delta \theta_{\text {Bench }}\right)$ by combining this mechanical deviation $\varepsilon$, with the difference between coil rotation axis and magnetic axis. Now by this procedure the right deviation of the magnetic axis with respect to the mechanical axis $\left(\Delta x_{\text {Magnet }}=x_{\text {meas }}+\Delta x_{\text {Bench }} ; \Delta y_{\text {Magnet }}=\right.$ $y_{\text {meas }}+\Delta y_{\text {Bench }} ; \Delta \theta_{\text {Magnet }}=\theta_{\text {meas }}+\Delta \theta_{\text {Bench }}$ ) can be generated.

\section{E. Accuracy of the Transfer Function Measurement}

Several systematic or random errors degrade the absolute measurement of the field/current absolute relationship (also called transfer function). A calibration is needed to know for instance the error induced in the transfer function due to the radial or the angular position and the opening angle of the coil. All the benches are calibrated with respect to each other in order to agree within about $0.6 \%$. 


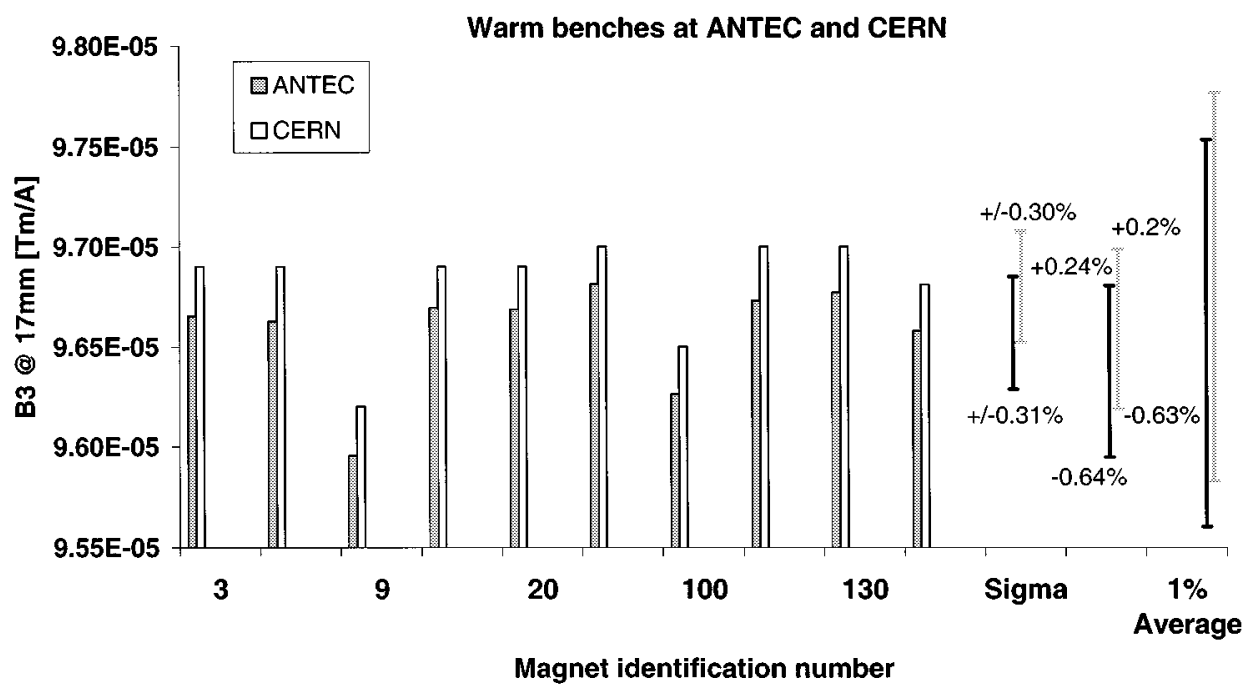

Fig. 3. Main field strength for 9 magnets measured at Antec and at CERN. The figure also shows the $1 \%$ range centered on the average of the main field strength.

\section{F. Measurement Procedure}

Deviations of the magnetic axis of more than $0.1 \mathrm{~mm}$ from the mechanical axis and/or angular deviations of more than 1.5 , 2, $2.5 \mathrm{mrad}$ twist of the magnetic field, respectively for MCS, MCD and MCO magnets, will cause the magnet to be rejected. The magnet field acceptance criteria specifies that all harmonics (skew and normal) must be below $1 \%$ of the main field at $17 \mathrm{~mm}$ radius corresponding to an axis offset of $0.1 \mathrm{~mm}$ and to a twist angle of 1.5, 2 and $2.5 \mathrm{mrad}$ following MCS, MCD and MCO magnet.

The system prints the two coordinates of the magnetic axis position and orientation of the main field with respect to the reference dowel holes in the mechanical case of the magnet, and multipoles calculated in the reference frame of that magnetic axis. All harmonics up to order 15 are displayed in Tm/A and reported at a reference radius of $17 \mathrm{~mm}$.

The automatic analysis provides verifications of the main magnet parameters discussed above, i.e., it prints and stores these results on-line.

\section{MAGNETiC MEASUREMENT Results}

In this section we present a comparison of the first measurements, performed using the CERN-built room temperature bench at ANTEC (Spain), on 9 pre-series MCS magnets, with those measurements on the same magnets made with an identical bench maintained at CERN.

\section{A. Main Field Component}

Fig. 3 shows the results for the main field (B3-MCS magnets). The difference in the average transfer function between these two sets of measurements is $0.3 \%$. Exchanging the two electronic racks on the same bench affects the main field at a level of $0.2 \%$. Further, there have been some indications that the coil calibration also drifts in the $0.1 \%$ range. Together, these two errors could account for the $0.3 \%$ difference in main field between the two benches. The differences between racks were not affected by swapping integrators. Part of the error may come from calibration of the current measurement, but in practice it
Warm benches at Antec and CERN

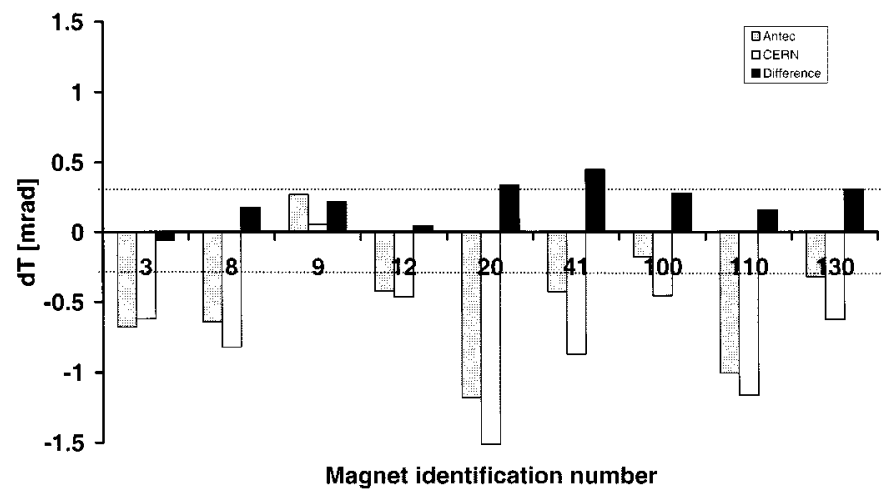

Fig. 4. Field orientation measured at Antec and CERN. The dotted line indicates the more pessimistic expected measurement error.

is difficult to adjust this to much better than $0.1 \%$. Also the stability of this adjustment is not known. The average transfer function in the two sets of measurements is measured at $1 \mathrm{~A}$ with the same standard deviation of $\approx 0.3 \%$ and approximately the same maximum range around the average of $-0.64 \%,+0.24 \%$ (ANTEC), and $-0.63 \%,+0.20 \%$ (CERN). Regarding magnetic field quality, the acceptable limit for harmonics other than $B 3$ is $10^{-6} \mathrm{Tm} / \mathrm{A}$, well above the difference between the two benches for harmonics up to order 15 (Maximum difference for $B 1=$ $\left.10^{-9} \mathrm{Tm} / \mathrm{A}\right)$.

\section{B. Field Orientation and Magnet's Axis Position}

The field orientation results are shown in Fig. 4, and the axis position errors are given in Figs. 5 and 6.

Also shown in these figures are the differences between the two benches. Note that in all these figures the full scale of the plots represents the tolerances specified for MCS magnets. The residual differences between the two sets of measurements should be compared with the uncertainty in the measurement which originates predominantly from the reproducibility in mechanical positioning of the magnet on the bench. For both benches the standard deviations in the reproducibility on the 


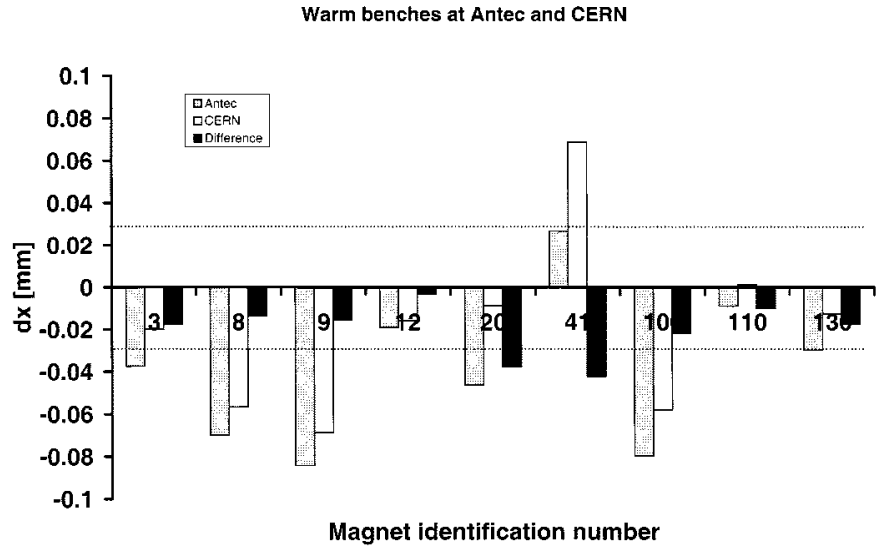

Fig. 5. Axis $d x$ offset measured at Antec and CERN. The dotted line indicates the more pessimistic expected measurement error.

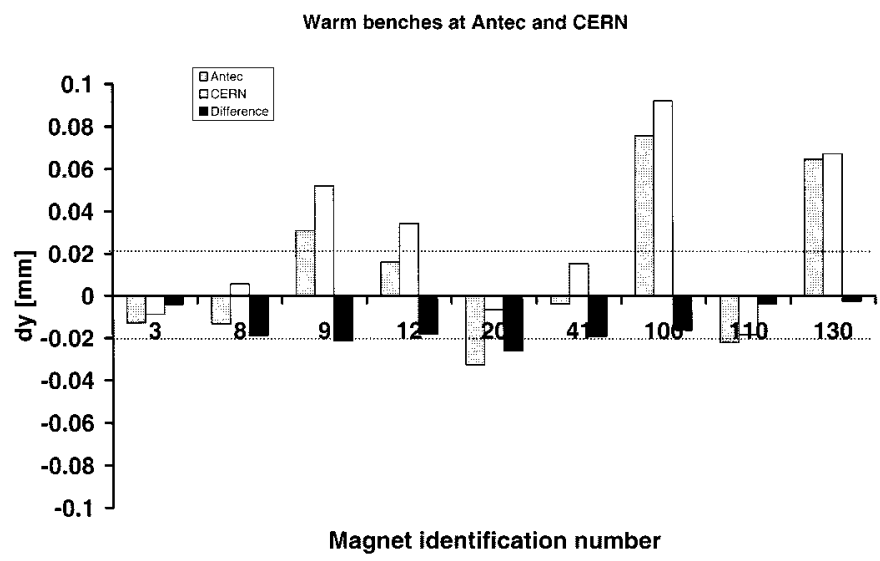

Fig. 6. Axis $d y$ offset measured at Antec and CERN. The dotted line indicates the more pessimistic expected measurement error.

magnetic axis position and field orientation measurements, taking repetitive mounting of the magnet on the bench into account, are evaluated at $\approx 5 \mu \mathrm{m}$ and $\approx 0.05 \mathrm{mrad}$ respectively, with reference holes drilled within tolerance $(6 H 7)$. Nevertheless we noticed that the standard deviations in the axis position and field orientation could be spread more largely (up to $d x \approx$ $10 \mu \mathrm{m}, d y \approx 7 \mu \mathrm{m}, d \theta \approx 0.1 \mathrm{mrad}$ ) depending on the deviation of the dowel holes diameter from this tolerance; i.e., accurate calibration of the bench and precise measurement become difficult for the following version. The reference holes drilled in the pre-series magnets cases were not precise enough due to difficulties introduced by the nickel plating. This uncertainty contributes to errors in the measurements via two channels. During calibration of the bench the magnet is installed in three different positions. Errors of up to twice the magnet positioning uncertainty can therefore enter the calibration, and subsequently appear as a systematic error in the measurement. The positioning uncertainty appears once again as a random error during the actual measurement. The final errors can increase by up to twice the systematic uncertainty plus once the random, for each bench. The differences between the two benches shown in Figs. 4-6 are therefore in reasonable line with expectations associated with the more pessimistic error in the measurement (dotted line in Figs. 4-6).

Comparison between measurements performed at CAT (India) and at CERN on MCS and MCDO magnets give similar results. The bench now at industry is producing results of the same quality as prior to delivery, and these results are adequate for testing the magnets to the specifications.

\section{CONCLUSIONS}

Good agreement is found between measurements made at magnet manufacturers and repeated at CERN with the CERNbuilt room temperature Industry bench. The results confirm successful installation and operation of the bench in industry.

The quality of measurements performed on this bench so far on the spool corrector magnets allows to maintain the basis of the previous test station so as to define the new generation of bench to measure the other corrector magnets (Lattice and Orbit correctors, Correctors for inner triplets ...) longer and bigger and with different mechanical reference points than the spool corrector magnets. These new benches are being manufactured at CERN.

\section{ACKNOWLEDGMENT}

The authors wish to acknowledge the advice of M. Allitt and A. Ijspeert during the prototyping phase of these corrector magnets, P. Viret and M. Ky for commissioning the measurement benches, and the encouragement of P. Sievers (CERN-LHCMTA).

\section{REFERENCES}

[1] Z. Ang et al., "Measurements of the LHC corrector magnets at room and cryogenic temperatures," in EPAC-98, Stockholm, Sweden, June 1998.

[2] J. Salminen et al., "Experience with the fabrication and testing of the sextupole superconducting corrector magnets for the LHC," in MT15, Bejing, Oct. 1997.

[3] L. Bottura, "Standard analysis procedures for field quality measurement of the LHC magnets-Part I: Harmonics," LHC Engineering Specification, LHC-XMT-ES-0001 rev 0.2, 2001.

[4] L. Madaro et al., "A VME-based Labview system for the magnet measurements of the LHC prototype dipoles," in EPAC-96, Sitges, Spain, June 1996.

[5] A. K. Jain, "Harmonic coils," in Proc. CERN Accelerator School (CAS), S. Turner, Ed. Geneva, 1998, pp. 175-218. 